\title{
7-Methylguanine Traps PARP-1 on Nucleosomes: spFRET Microscopy Study
}

\author{
Natalya Maluchenko ${ }^{1}$, Dmitry Nilov ${ }^{2}$, Alexey Feofanov ${ }^{1,3}$, Alexandra Lys ${ }^{1 *}$, Mikhail Kutuzov ${ }^{4}$, \\ Nadezhda Gerasimova ${ }^{1}$ and Vasily Studitsky ${ }^{5}$ \\ 1. Faculty of Biology, Lomonosov Moscow State University, Moscow, Russia. \\ 2.Belozersky Institute of Physicochemical Biology, Lomonosov Moscow State University, Moscow, \\ Russia. \\ 3. Shemyakin-Ovchinnikov Institute of Bioorganic Chemistry, Russian Academy of Sciences, Moscow, \\ Russia. \\ 4. Institute of Chemical Biology and Fundamental Medicine, Siberian Branch, Russian Academy of \\ Sciences, Novosibirsk, Russia. \\ 5. Fox Chase Cancer Center, Philadelphia, USA. \\ * Corresponding author: lys-alex-bio-msu@yandex.ru
}

The poly (ADP-ribose) polymerase 1 (PARP-1) is a promising molecular target for the antitumor drug development. Overexpression or loss of PARP-1 leads to disturbances in the process of DNA repair and transcription of genes involved in DNA replication and cell cycle regulation and, as a consequence, to general genome instability. Increased PARP-1 activity is observed in numerous cancers [1,2], and considered as a possible mechanism of resistance to DNA-damaging therapeutics [3]. A high level of PARP-1 expression correlates with a more aggressive phenotype of cancers [4]. Many PARP-1 inhibitors are undergoing clinical trials, and four of them have been already approved for the treatment of cancer patients. These inhibitors are thought to exert their cytotoxicity through both inhibition of catalytic activity and trapping of DNA-PARP-1 complexes [5,6]. Trapping of PARP-1 on DNA and formation of non-productive complexes was proved to be a more effective way to kill cancer cells than simple inhibition of PARP-1 activity. However, in the nuclei PARP-1 proteins interact with DNA organized into chromatin, and contribution of chromatin structure to PARP-1 trapping requires detailed investigation. Here we applied single-particle Förster resonance energy transfer (spFRET) microscopy (similar as described elsewhere [7-9]) to analyze PARP-1 trapping on nucleosomal templates by a new PARP-1 inhibitor 7-methylguanine (7-MG, Fig. 1 A), which is able to accelerate apoptotic death of BRCA1-deficient breast cancer cells induced by cisplatin and doxorubicin [10].

Mononucleosomes are a convenient "minimal" experimental system mimicking the chromatin packing of DNA. To make mononucleosomes suitable for structural studies using spFRET microscopy, a pair of labels (donor, Cy3, and acceptor, Cy5) was introduced into neighboring gyres of nucleosomal DNA in positions $+13 /+91$ bp relative to the beginning of nucleosomal DNA (Fig. $1 \mathrm{~B}$ ). Such labeling provides efficient FRET between the fluorophores without disturbance of histone-DNA contacts in the assembled nucleosomes [9]. Analysis of spFRET in mononucleosomes revealed two structurally different subpopulations of nucleosomes: a major subpopulation with high FRET values (high-FRET), and a minor subpopulation with low FRET values (low-FRET) (Fig. 1 B). Formation of nucleosome-PARP-1 complexes is characterized by: a) the narrowing of the high-FRET peak; b) appearance of a new intermediate-FRET peak; c) disappearance of the low-FRET peak (Fig $1 \mathrm{C}$ ). After adding PARP-1 substrate NAD + to the nucleosome-PARP-1 complexes a recovery of the original nucleosome structure occurs indicating dissociation of the complex during ADP-ribosylation (Fig $1 \mathrm{C}$ ). Our study revealed that 7-MG does not affect the structure of free nucleosomes, but enhances PARP-1 binding to 
nucleosomes (Figs. 1 C, D). Moreover, 7-MG blocks NAD-induced dissociation of PARP-1 from the complexes with nucleosomes, thus trapping the enzyme on nucleosomes (Figs. 1 D).

In conclusion, 7-MG inhibits PARP-1, enhances formation of enzyme-nucleosome complexes and traps PARP-1 in these complexes that can be a basis of its anticancer activity [11].

References:

[1] M Rodríguez et al., PLoS Genet. 9 (2013), p. e1003531.

[2] S Nowsheen et al., PLoS One 7 (2012), p. e46614.

[3] W Wang et al., Cancers (Basel) 11 (2019), p. e43.

[4] P Domagala et al., Breast Cancer Res. Treat 127 (2011), p. 861.

[5] T Hopkins et al., Mol. Cancer Res.17 (2019), p. 409.

[6] M Langelier et al., Nat. Commun. 9 (2018), p. 844.

[7] D Sultanov et al., AIMS Genetics 4 (2017), p. 21.

[8] M Valieva et al., Nat. Struct. Mol. Biol. 23 (2016), p. 1111.

[9] K Kudryashova et al., Methods Mol. Biol. 1288 (2015), p. 395.

[10] D Nilov et al., Acta Naturae 8 (2016), p. 108.

[11] Financial supported by RFBR grant № 17-00-00163 (17-00-00132, 17-00-00097) is acknowledged.

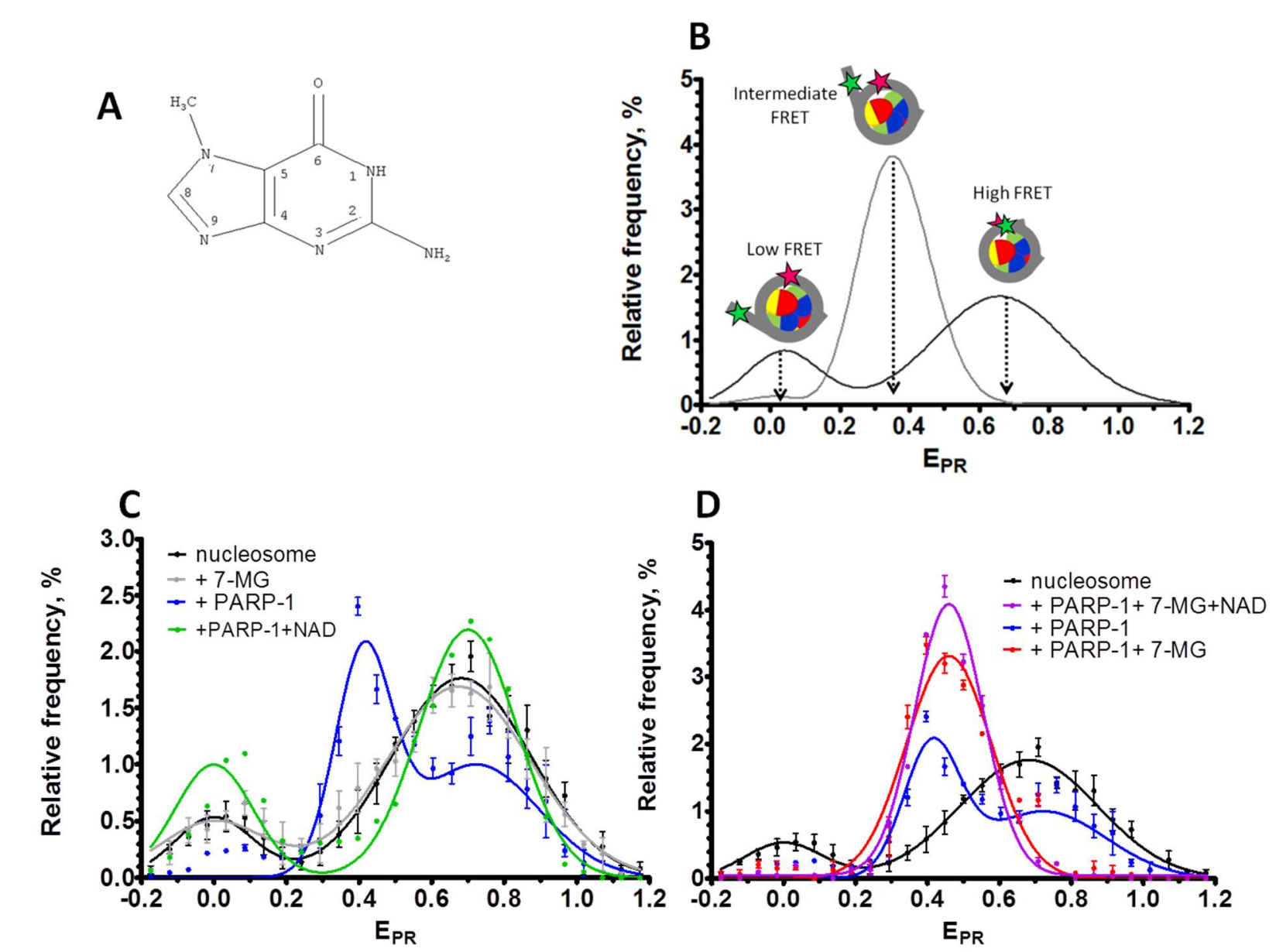

Figure 1. spFRET microscopy analysis of interactions between nucleosomes, PARP-1, NAD+ and 7-MG. (A) Structure of 7-MG. (B) The experimental approach for analysis of PARP-1-dependent changes in nucleosome structure on the basis of spFRET microscopy. (C, D) Histograms showing relative frequency distributions of nucleosomes by FRET values (EPR) in the presence of different compounds. Concentrations: $1 \mathrm{nM}$ nucleosomes, $20 \mathrm{nM}$ PARP-1, $450 \mu \mathrm{M} 7-\mathrm{MG}, 100 \mu \mathrm{M}$ NAD+. 